\title{
Studies on Anti-Inflammation Activity of Phenols Using Newly Introduced Balaban Indices
}

\author{
Dipika Sharma ${ }^{1}$, Priyanka Mukadam ${ }^{2}$ \\ School of Chemistry and Biochemistry, Vikram University, Ujjain, India
}

\begin{abstract}
The interated 'sum' versus 'product' operation concept recently introduced by Balaban-KhadikarSufia yielding newly introduced $F$ and $G$ indices, has been used for proposing most significant QSAR model for modeling anti inflammatory activity of phenol. Results are discussed on the basis of well known statistical parameters.
\end{abstract}

Keywords: QSAR, anti inflammatory activity, phenols

\section{Introduction}

Inflammation is a complex and dynamic biological process generally described as the characteristic response of animal tissue to injury. Certain coordinal signs of inflammation namely redness, heat, swelling and pain were clearly recognized [1-2].The search for an anti-inflammatory remedy in modern time was masked by the introduction of sulicin for the treatment of acute rheumatism. During the last-two decade's corticosteroids and non-steroidal agents have provided much relief to suffer of the disorders caused by inflammation.

New drugs with still better relief giving potential are synthesized applying the application of mathematical concept called graph theory and topology [3]. All such new compounds are possible to prepare applying combinatory and throughout chemistry [4]. Such new synthesis is possible by the correlation analysis, in that correlation is investigated by correlating the activity with topological indices5. Needless to state that topological index is a numerical representation of molecular structure [5-6]. Hence, quantitative structureactivity relationship (QSAR) is only possible using topological indices as molecular descriptors. In the present study we have attempted QSAR study on the modeling of anti-inflammatory activity of phenols (Table 1) using regression analysis. In view of this we have for the first-time used the new topological indices developed by Balaban-Khadikar-Sufia. These indices ( $F$ and $G$ ) are the 'Shape' indices and therefore we can use them for investigating the influence of 'Shape' on anti-inflammatory activity. In addition, we have also used the wellknown Balaban $\mathbf{J}$ index [7-10]. This $\mathbf{J}$ index is supposed to be most discriminating index known and developed in chemical graph theory.

\section{Result And Discussion}

J Index:

While discussing interated 'sum' versus 'product' operations Balaban-Khadikar-Sufia has introduced two new Balaban type indices viz $\mathrm{F}$ and $\mathrm{G}$ using the expression of Balaban $\mathrm{J}$ index:

$$
\mathbf{J}=(\mathrm{E} / \mathrm{R}+1) \sum(\text { di.dj })^{-1 / 2} \quad \text { eql edgs } \quad \ldots . . .(1)
$$

Where $\mathrm{E}$ is the number of edges, di and dj are the distance sums and $\mathrm{R}$ is cyclomatic number defined by the following expression

$$
\mathrm{R}=\mathrm{E}-n+1
$$

Where $n$ is the number vertex (atoms) in the molecular graph.

The two new indices $\mathrm{F}$ and $\mathrm{G}$ are defined by the following expression.

F Index:

$$
\begin{aligned}
\mathbf{F} & =\mathbf{E} \sum(\mathbf{d i d} \cdot \mathbf{d j})^{-1 / 2} \\
& =\mathbf{J}(\mathbf{R}+1)
\end{aligned}
$$

G Index:

$$
\begin{aligned}
\mathrm{G} & =\left[n^{2} \mathrm{E} / n+\mathrm{R}+1\right] \sum(\mathrm{di} . \mathrm{dj})^{-1 / 2} \\
& =\left[n^{2} \mathrm{~F} / n+\mathrm{R}+1\right]
\end{aligned}
$$

Correlation Coefficient:

It is a correlation between the observed value of the variable on one side and its estimated value expressed in terms of the dependent variables on the other. It is a relative measure of the quality of fit of the model. Its value depends on the overall variance of the data. It is denoted by $r$.

Coefficient of determination:

The square of correlation coefficient, i.e. $r^{2}$, which is also known as coefficient of determination is used for interpreting the value of coefficient of correlation between two set of variables. 


\section{F-Test:}

F-test tool of statistical analysis is given by R.A. Fisher. This parameter is one of several variance related parameters that can be used to compare two models differing by one or more variables. This statistics is used to determine whether a more complex model is significantly better than a less complex one.

$$
\mathrm{F}=\frac{r^{2}(\mathrm{~N}-k-1)}{k\left(1-r^{2}\right)}
$$

Where $\mathrm{N}$ is number of sample, $k$ is number of descriptors and $r$ is regression coefficient

Adjusted r-squared:

$r_{A}^{2}$ is an adjusted version of $r^{2}$, the adjustment seeks to remove the distortion due to small sample size.

$$
r_{A}^{2}=1-\frac{(\mathrm{N}-1)\left(1-r^{2}\right)}{(\mathrm{N}-k-1)}
$$

Where $\mathrm{N}$ is number of sample $k$ is number of descriptors and $r$ is regression coefficient.

The values of $\mathrm{G}$ along with the value of $-\log 1 / \mathrm{IC}_{50}$ are presented in Table 1 for the phenols used in the present investigation. The symbol $\mathrm{N}$ needed for modeling while using NCSS software given in table 1.

After performing regression analysis we get two model one variable \& two variables, which are given below:

One-variable model obtained is as below:

Anti-inflammatory activity $\left(-\log 1 / \mathrm{IC}_{50}\right)=5.7425-0.1134( \pm 0.0161)(\mathrm{G})$

$\mathrm{N}=17, c v=0.1698, r^{2}=0.7678, r_{A}^{2}=0.7524, \mathrm{~F}=49.613$

Where $\mathrm{N}=$ Number of sample, $c v=$ Coefficient of Variation, $r^{2}=$ Coefficient of determination, $r_{A}^{2}=$ Adjusted $r$-squared, $\mathrm{F}=\mathrm{F}$-Test

Two-variable model obtained is as below:

Anti-inflammatory activity $\left(-\log 1 / \mathrm{IC}_{50}\right)=3.7615+1.1928( \pm 2.4733)(\mathrm{J})-0.1372( \pm 0.0521)(\mathrm{G})$

eq..... (8)

$\mathrm{N}=17, c v=0.1743, r^{2}=0.7716, r_{A}^{2}=0.7390, \mathrm{~F}=23.654$

Where, $\mathrm{N}=$ Number of sample, $c v=$ Coefficient of Variation, $r^{2}=$ Coefficient of determination, $r_{A}^{2}=$ Adjusted $r$-squared

There are only two statistically significant models shown in equation 7 and 8 , out of which the second model which shown by equation 8 gives better $r^{2}=0.7716$. This model is a two-variable model containing $\mathrm{J}$ and $\mathrm{G}$ as the correlating parameters. The onevariable model containing $\mathrm{G}$ as the correlating parameter yield $r^{2}=0.7678$. A careful examination of this indicates that as we go from further from one-variable model (equation no. 7) to two-variable model (equations no 8 ) the $r^{2}$ value changes from 0.7524 to 0.7390 . That is $r_{A}^{2}$ value decreases when $\mathrm{J}$ is added to one-variable model (equation no. 7) containing $\mathrm{G}$ as the correlating parameter. It clearly indicates that addition of $\mathbf{J}$ is not fully justified and that the one-variable model is the most appropriate model for modeling antiinflammatory activity of phenols.

The data obtained from indicates that the above model is free from the defect due to colinearity. Also, that $r_{\text {Pred }}^{2}=0.7678$ indicates that the proposed model shows $76.78 \%$ variation in the anti-inflammatory activity of phenols.

\begin{tabular}{|c|c|c|c|}
\hline S1 & $\mathrm{R}$ & G & $-\log 1 / 1 C_{50}$ \\
\hline 1 & $\mathrm{H}$ & 23.117 & 3.204 \\
\hline 2 & 2-chloro- & 29.171 & 2.441 \\
\hline 3 & 2-bromo- & 29.171 & 2.875 \\
\hline 4 & 2-methyl- & 29.171 & 2.176 \\
\hline 5 & 2-ethyl- & 33.829 & 1.869 \\
\hline 6 & 2-methoxy- & 33.829 & 1.869 \\
\hline 7 & 3-methy1- & 28.557 & 2.786 \\
\hline 8 & 3-methoxy- & 32.871 & 1.754 \\
\hline 9 & 4-methy1- & 28.058 & 2.12 \\
\hline 10 & 2,3-di-methyl- & 35.537 & 1.806 \\
\hline 11 & 2,4-di-methyl- & 34.550 & 2.568 \\
\hline 12 & 2,5-di-methyl- & 34.550 & 1.653 \\
\hline 13 & 2,6-di-methyl- & 35.537 & 1.203 \\
\hline 14 & 3,5-di-methyl- & 34.477 & 1.806 \\
\hline 15 & 2,3,6-tri-methyl- & 41.933 & 0.903 \\
\hline 16 & 2,3,5-tri-methyl- & 41.450 & 1.431 \\
\hline 17 & 2,4,6-tri-methyl- & 41.450 & 0.845 \\
\hline
\end{tabular}

\section{Figures And Tables}

Table 1: The phenols and their anti-inflammatory activity $(-\log 1 / \mathrm{IC} 50)$ 


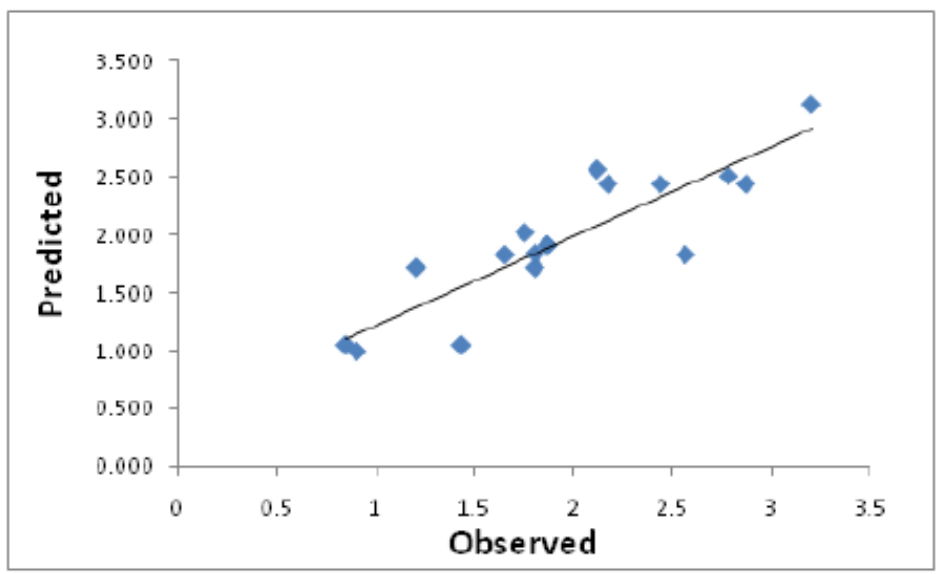

Fig 1:-. Correlation of observed and predicted anti-inflammatory activity using one-variable model

\section{Conclusion}

The results obtained indicate that the newly introduced $\mathrm{G}$ index can be successfully used for modeling anti-inflammatory activity of phenols. The negative coefficient of $\mathrm{G}$ indicates that the lowering of shape increases anti-inflammatory activity of phenols.

\section{Acknowledgement}

The authors are thankful to Professor B.K. Mehta (Vikram Universi ty) \& Late Dr. P.V. Khadikar (DAVV) for providing necessary guidance.

\section{Reference}

[1]. Topliss, J.G.; Edward, R.P. Chance factor in studies of quantitative Structure Activity Relationships. J. Med. Chem., 1979, 22, pp.1238-1244.

[2]. $\quad$ Minailiuc, O.M.; Katona, G.; Diudea, M.V.; Strunje, M.; Graovac, A.; Gutman, I. Szeged Fragmental Indices. Croatica Chemica Acta, 1998, 71 (3), pp.473-488

[3]. Kier, L.B.; Hall, L.H. The nature of structure-activity relationships and their relation to molecular connectivity. Eur. J. Med. Chem., 1977, 12, pp.307-312.

[4]. Rabinovitz, J.L.; Myerson, R.M.; Topics in Medicinal Chemistry, Wiley, New York, 1967

[5]. Gutman, I.; Klavzar, S. An algorithm for the calculation of the Szeged index of benzenoid hydrocarbons. Journal of Chemical Information and Computer Sciences, 1995, 35 (6), pp.1011-1014.

[6]. Pogliani, L. From molecular connectivity indices to semiempirical connectivity terms: Recent trends in graph theoretical descriptors. Chemical Reviews, 2000, 100 (10), pp. 3827-3858.

[7]. Sharma, V.; Karmarkar, S.; Thakral, G.S.; Khadikar, P.V. Estimation of the anti-inflammatory activity of phenols by Szeged index (Sz). Nat. Acad. Sci. Letters, 2000, 23, pp. 5-6.

[8]. Khadikar, P.V.; Phadnis, A.; Shrivastava, A. QSAR study on toxicity to aqueous organisms using the PI index. Bioorganic and Medicinal Chemistry, 2002, 10 (4), pp. 1181-1188.

[9]. Balaban, A.T.; Khadikar, P.V.; Aziz, S. Comparison of Topological Indices Based on Iterated 'Sum' versus 'Product' Operations. Iranian. J. Math. Chem., 2010, 1, pp. 43-60.

[10]. Balaban, A.T. Highly discriminating distance-based topological index. Chem. phys. Lett., 1982, 80, pp. 399-404. 